\title{
A Matlab Program to Calculate the Maximum Entropy Distributions
}

\author{
Ali Mohammad-Djafari \\ Laboratoire des Signaux et Systèmes (CNRS-ESE-UPS) \\ École Supérieure d'Électricité \\ Plateau de Moulon, 91192 Gif-sur-Yvette Cédex, France
}

\begin{abstract}
The classical maximum entropy (ME) problem consists of determining a probability distribution function (pdf) from a finite set of expectations $\mu_{n}=\mathrm{E}\left\{\phi_{n}(x)\right\}$ of known functions $\phi_{n}(x), n=0, \ldots, N$. The solution depends on $N+1$ Lagrange multipliers which are determined by solving the set of nonlinear equations formed by the $N$ data constraints and the normalization constraint. In this short communication we give three Matlab programs to calculate these Lagrange multipliers. The first considers the case where $\phi_{n}(x)$ can be any functions. The second considers the special case where $\phi_{n}(x)=x^{n}, n=0, \ldots, N$. In this case the $\mu_{n}$ are the geometrical moments of $p(x)$. The third considers the special case where $\phi_{n}(x)=\exp (-j n \omega x), n=0, \ldots, N$. In this case the $\mu_{n}$ are the trigonometrical moments (Fourier components) of $p(x)$. We give also some examples to illustrate the usefullness of these programs.
\end{abstract}

\section{Introduction}

Shannon (1948) indicated how maximum entropy (ME) distributions can be derived by a straigtforward application of the calculus of variations technique. He defined the entropy of a probability density function $p(x)$ as

$$
H=-\int p(x) \ln p(x) \mathrm{d} x
$$

Maximizing $H$ subject to various side conditions is well-known in the literature as a method for deriving the forms of minimal information prior distributions; e.g. Jaynes (1968) and Zellner (1977). Jaynes (1983) has extensively analyzed examples in the discrete case, while in Lisman and Van Znylen (1972), Rao (1973) and Gokhale (1975), Kagan, Linjik continuous cases are considered. In the last case, the problem, in its general form, is the following

$$
\begin{aligned}
\operatorname{maximize} \quad H & =-\int p(x) \ln p(x) \mathrm{d} x \\
\text { subject to } \mathrm{E}\left\{\phi_{n}(x)\right\} & =\int \phi_{n}(x) p(x) \mathrm{d} x=\mu_{n}, \quad n=0, \ldots, N
\end{aligned}
$$

where $\mu_{0}=1, \phi_{0}(x)=1$ and $\phi_{n}(x), n=0, \ldots, N$ are $N$ known functions, and $\mu_{n}, n=$ $0, \ldots, N$ are the given expectation data. The classical solution of this problem is given by

$$
p(x)=\exp \left[-\sum_{n=0}^{N} \lambda_{n} \phi_{n}(x)\right]
$$


The $(N+1)$ Lagrange parameters $\boldsymbol{\lambda}=\left[\lambda_{0}, \ldots, \lambda_{n}\right]$ are obtained by solving the following set of $(N+1)$ nonlinear equations

$$
G_{n}(\boldsymbol{\lambda})=\int \phi_{n}(x) \exp \left[-\sum_{n=0}^{N} \lambda_{n} \phi_{n}(x)\right] \mathrm{d} x=\mu_{n}, \quad n=0, \ldots, N
$$

The distributions defined by (3) form a great number of known distributions which are obtained by choosing the appropriate $N$ and $\phi_{n}(x), n=0, \ldots, N$. In general $\phi_{n}(x)$ are either the powers of $x$ or the logarithm of $x$. See Mukhrejee and Hurst (1984), Zellner (1988), Mohammad-Djafari (1990) for many other examples and discussions. Special cases have been extensively analyzed and used by many authors. When $\phi_{n}(x)=x^{n}, n=0, \ldots, N$ then $\mu_{n}, n=0, \ldots, N$ are the given $N$ moments of the distribution. See, for example, Zellner (1988) for a numerical implementation in the case $N=4$.

In this communication we propose three programs written in MATLAB to solve the system of equations (4). The first is a general program where $\phi_{n}(x)$ can be any functions. The second is a special case where $\phi_{n}(x)=x^{n}, n=0, \ldots, N$. In this case the $\mu_{n}$ are the geometrical moments of $p(x)$. The third is a special case where $\phi_{n}(x)=\exp (-j n \omega x), n=$ $0, \ldots, N$. In this case the $\mu_{n}$ are the trigonometrical moments (Fourier components) of $p(x)$. We give also some examples to illustrate the usefullness of these programs.

\section{Principle of the method}

We have seen that the solution of the standard ME problem is given by (3) in which the Lagrange multipliers $\boldsymbol{\lambda}$ are obtained by solving the nonlinear equations (4). In general, these equations are solved by the standard Newton method which consists of expanding $G_{n}(\boldsymbol{\lambda})$ in Taylor's series around trial values of the lambda's, drop the quadratic and higher order terms, and solve the resulting linear system iteratively. We give here the details of the numerical method that we implemented. When developing the $G_{n}(\boldsymbol{\lambda})$ in equations (4) in first order Taylor's series around the trial $\lambda^{0}$, the resulting linear equations are given by

$$
G_{n}(\boldsymbol{\lambda}) \cong G_{n}\left(\boldsymbol{\lambda}^{0}\right)+\left(\boldsymbol{\lambda}-\boldsymbol{\lambda}^{0}\right)^{t}\left[\operatorname{grad} G_{n}(\boldsymbol{\lambda})\right]_{\left(\boldsymbol{\lambda}=\boldsymbol{\lambda}^{0}\right)}=\mu_{n}, \quad n=0, \ldots, N
$$

Noting the vectors $\boldsymbol{\delta}$ and $\boldsymbol{v}$ by

$$
\begin{gathered}
\boldsymbol{\delta}=\boldsymbol{\lambda}-\boldsymbol{\lambda}^{0} \\
\boldsymbol{v}=\left[\mu_{0}-G_{0}\left(\boldsymbol{\lambda}^{0}\right), \ldots, \mu_{N}-G_{N}\left(\boldsymbol{\lambda}^{0}\right)\right]^{t}
\end{gathered}
$$

and the matrix $\boldsymbol{G}$ by

$$
\boldsymbol{G}=\left(g_{n k}\right)=\left(\frac{\partial G_{n}(\boldsymbol{\lambda})}{\partial \lambda_{k}}\right)_{\left(\boldsymbol{\lambda}=\boldsymbol{\lambda}^{0}\right)} n, k=0, \ldots, N
$$

then equations (5) become

$$
\boldsymbol{G} \delta=\boldsymbol{v}
$$

This system is solved for $\boldsymbol{\delta}$ from which we drive $\boldsymbol{\lambda}=\boldsymbol{\lambda}^{0}+\boldsymbol{\delta}$, which becomes our new initial vector $\lambda^{0}$ and the iterations continue until $\delta$ becomes appropriately small. Note that the matrix $\boldsymbol{G}$ is a symmetric one and we have

$$
g_{n k}=g_{k n}=-\int \phi_{n}(x) \phi_{k}(x) \exp \left[-\sum_{n=0}^{N} \lambda_{n} \phi_{n}(x)\right] \mathrm{d} x \quad n, k=0, \ldots, N
$$


So in each iteration we have to calculate the $N(N-1) / 2$ integrals in the equation (8). The algorithm of the general Maximum Entropy problem is then as follows:

1. Define the range and the discretization step of $x$

$(x \min , x \max , d x)$.

2. Write a function to calculate $\phi_{n}(x), n=0, \ldots, N$

$\left(f i n \_x\right)$.

3. Start the iterative procedure with an initial estimate $\boldsymbol{\lambda}^{0}$

(lambda0).

4. Calculate the $(N+1)$ integrals in equations (4) and the $N(N-1) / 2$ distinct elements $g_{n k}$ of the matrix $\boldsymbol{G}$ by calculating the integrals in the equations(8) (Gn, gnk).

5. Solve the equation (7) to find $\delta$

(delta).

6. Calculate $\boldsymbol{\lambda}=\boldsymbol{\lambda}^{0}+\boldsymbol{\delta}$ and go back to step 3 until $\boldsymbol{\delta}$ becomes negligible.

The calculus of the integrals in equations (4) and (8) can be made by a univariate Simpson's method. We have used a very simplified version of this method.

\section{Case of geometrical moments}

Now consider the special case of moments problem where $\phi_{n}(x)=x^{n}, \quad n=0, \ldots, N$. In this case equations (3), (4) and (8) become

$$
\begin{gathered}
p(x)=\exp \left[-\sum_{m=0}^{N} \lambda_{m} x^{m}\right] \\
G_{n}(\boldsymbol{\lambda})=\int x^{n} \exp \left[-\sum_{m=0}^{N} \lambda_{m} x^{m}\right] \mathrm{d} x=\mu_{n}, \quad n=0, \ldots, N \\
g_{n k}=g_{k n}=-\int x^{n} x^{k} \exp \left[-\sum_{m=0}^{N} \lambda_{m} x^{m}\right] \mathrm{d} x=-G_{n+k}(\boldsymbol{\lambda}) \quad n, k=0, \ldots, N
\end{gathered}
$$

This means that the $[(N+1) \times(N+1)]$ matrix $\boldsymbol{G}$ in equation (7) becomes a symmetric Hankel matrix which is entirely defined by $2 N+1$ values $G_{n}(\boldsymbol{\lambda}), n=0, \ldots, 2 N$. So the algorithm in this case is the same as in the precedent one with two simplifications

1. In step 2 we do not need to write a seperate function to calculate the functions $\phi_{n}(x)=$ $x^{n}, n=0, \ldots, N$.

2. In step 4 the number of integral evaluations is reduced, because the elements $g_{n k}$ of the matrix $\boldsymbol{G}$ are related to the integrals $G_{n}(\boldsymbol{\lambda})$ in equations (10). This matrix is defined entirely by only $2 N+1$ components.

\section{Case of trigonometrical moments}

Another interesting special case is the case where the data are the Fourier components of $p(x)$

$$
\mathrm{E}\left\{\exp \left(-j n \omega_{0} x\right)\right\}=\int \exp \left(-j n \omega_{0} x\right) p(x) \mathrm{d} x=\mu_{n}, \quad n=0, \ldots, N
$$

where $\mu_{n}$ may be complex-valued and has the property $\mu_{-n}=\mu_{n}$. This means that we 
have the following relations

$$
\begin{aligned}
\phi_{n}(x) & =\exp \left(-j n \omega_{0} x\right), \quad n=-N, \ldots, 0, \ldots N, \\
p(x) & =\exp \left[-\operatorname{Real} \sum_{n=0}^{N} \lambda_{n} \exp \left(-j n \omega_{0} x\right)\right], \\
G_{n}(\boldsymbol{\lambda}) & =\int \exp \left(-j n \omega_{0} x\right) p(x) \mathrm{d} x, \quad n=0, \ldots, N, \\
g_{n k} & =\left\{\begin{array}{ll}
-G_{n-k}(\boldsymbol{\lambda}) & \text { for } n \geq k, \\
-G_{n+k}(\boldsymbol{\lambda}) & \text { for } n<k
\end{array} \quad n, k=0, \ldots, N,\right.
\end{aligned}
$$

so that all the elements of the matrix $\boldsymbol{G}$ are related to the discrete Fourier transforms of $p(x)$. Note that $\boldsymbol{G}$ is a Hermitian Toeplitz matrix.

\section{Examples and Numerical Experiments}

To illustrate the usefullness of the proposed programs we consider first the case of the Gamma distribution

$$
p(x ; \alpha, \beta)=\frac{\beta^{(1-\alpha)}}{\Gamma(1-\alpha)} x^{\alpha} \exp (-\beta x), \quad x>0, \alpha<1, \beta>0 .
$$

This distribution can be considered as a ME distribution when the constraints are

$$
\begin{aligned}
\int p(x ; \alpha, \beta) \mathrm{d} x & =1 & \text { normalization } & \phi_{0}(x)=1, \\
\int x p(x ; \alpha, \beta) \mathrm{d} x & =\mu_{1} & \phi_{1}(x) & =x, \\
\int \ln (x) p(x ; \alpha, \beta) \mathrm{d} x & =\mu_{2} & \phi_{2}(x) & =\ln (x) .
\end{aligned}
$$

This is easy to verify because the equation (12) can be written as

$$
\begin{gathered}
p(x ; \alpha, \beta)=\exp \left[-\lambda_{0}-\lambda_{1} x-\lambda_{2} \ln (x)\right] \\
\text { with } \quad \lambda_{0}=-\ln \frac{\beta^{(1-\alpha)}}{\Gamma(1-\alpha)}, \quad \lambda_{1}=\beta \quad \text { and } \quad \lambda_{2}=-\alpha .
\end{gathered}
$$

Now consider the following problem : Given $\mu_{1}$ and $\mu_{2}$ determine $\lambda_{0}, \lambda_{1}$ and $\lambda_{2}$.

This can be done by the standard ME method. To do this, first we must define the range of $x,(x \min , \operatorname{xmax}, \mathrm{dx})$, and write a function $\mathrm{f}$ in_ $\mathrm{x}$ to calculate the functions $\phi_{0}(x)=1$, $\phi_{1}(x)=x$ and $\phi_{2}(x)=\ln x$ (See the function fin1_x in Annex). Then we must define an initial estimate $\boldsymbol{\lambda}^{0}$ for $\boldsymbol{\lambda}$ and, finally, let the program works.

The case of the Gamma distribution is interesting because there is an analytic relation between $(\alpha, \beta)$ and the mean $m=\mathrm{E}\{x\}$ and variance $\sigma^{2}=\mathrm{E}\left\{(x-m)^{2}\right\}$ which is

$$
\left\{\begin{array}{l}
m=(1-\alpha) / \beta \\
\sigma^{2}=(1-\alpha) / \beta^{2}
\end{array}\right.
$$


or inversely

$$
\left\{\begin{array}{l}
\alpha=\left(\sigma^{2}-m^{2}\right) / \sigma^{2} \\
\beta=m / \sigma^{2}
\end{array}\right.
$$

so that we can use these relations to determine $m$ and $\sigma^{2}$. Note also that the corresponding entropy of the final result is a byproduct of the function. Table (1) gives some numerical results obtained by ME_DENS1 program (See Annex).

Table 1.

\begin{tabular}{|cccccc|}
\hline$\mu_{1}$ & $\mu_{2}$ & $\alpha$ & $\beta$ & $m$ & $\sigma^{2}$ \\
\hline 0.2000 & -3.0000 & 0.2156 & -3.0962 & 0.2533 & 0.0818 \\
0.2000 & -2.0000 & -0.4124 & -6.9968 & 0.2019 & 0.0289 \\
0.3000 & -1.5000 & -0.6969 & -5.3493 & 0.3172 & 0.0593 \\
\hline
\end{tabular}

The next example is the case of a quartic distribution

$$
p(x)=\exp \left[-\sum_{n=0}^{4} \lambda_{n} x^{n}\right] .
$$

This distribution can be considered as a ME distribution when the constraints are

$$
\mathrm{E}\left\{x^{n}\right\}=\int x^{n} p(x) \mathrm{d} x=\mu_{n}, \quad n=0, \ldots, 4 \quad \text { with } \quad \mu_{0}=1 .
$$

Now consider the following problem: Given $\mu_{n}, n=1, \ldots, 4$ calculate $\lambda_{n}, n=0, \ldots, 4$. This can be done by the ME_DENS2 program. Table (2) gives some numerical results obtained by this program:

\begin{tabular}{|lllllllll|}
\hline \multicolumn{1}{c}{ Table 2.} \\
\hline$\mu_{1}$ & $\mu_{2}$ & $\mu_{3}$ & $\mu_{4}$ & $\lambda_{0}$ & $\lambda_{1}$ & $\lambda_{2}$ & $\lambda_{3}$ & $\lambda_{4}$ \\
0 & 0.2 & 0.05 & 0.10 & 0.1992 & 1.7599 & 2.2229 & -3.9375 & 0.4201 \\
0 & 0.3 & 0.00 & 0.15 & 0.9392 & 0.000 & -3.3414 & 0.0000 & 4.6875 \\
0 & 0.3 & 0.00 & 0.15 & 0.9392 & 0.000 & -3.3414 & 0.0000 & 4.6875 \\
\hline
\end{tabular}

These examples show how to use the proposed programs. A third example is also given in Annex which shows how to use the ME_DENS3 program which considers the case of trigonometric moments.

\section{Conclusions}

In this paper we addressed first the class of ME distributions when the available data are a finite set of expectations $\mu_{n}=\mathrm{E}\left\{\phi_{n}(x)\right\}$ of some known functions $\phi_{n}(x), n=0, \ldots, N$. We proposed then three Matlab programs to solve this problem by a Newton-Raphson method in general case, in case of geometrical moments data where $\phi_{n}(x)=x^{n}$ and in case of trigonometrical moments where $\phi_{n}(x)=\exp \left(-j n \omega_{0} x\right)$. Finally, we gave some numerical results for some special examples who show how to use the proposed programs. 
Ali Mohammad-Djafari

\section{REFERENCES}

[1] A. Zellnerr and R. Highfiled, "Calculation of Maximum Entropy Distributions and Approximation of Marginal Posterior Distributions, " Journal of Econometrics 37, 1988, 195-209, North Holland.

[2] D. Mukherjee and D.C. Hurst, "Maximum Entropy Revisited, "Statistica Neerlandica 38, 1984, n 1, 1-12.

[3] Verdugo Lazo and P.N. Rathie, "On the Entropy of Continuous Probability Distributions, " IEEE Trans., vol. IT-24, n 1, 1978.

[4] Gokhale, "Maximum Entropy Characterizations of some distributions, " Statistical distributions in Scientific work, vol. 3, 299-304 (G.P. Patil et al., Eds., Reidel, Dordrecht, Holland, 1975).

[5] Jaynes, "Papers on probability, statistics and statistical physics, " Reidel Publishing Company, Dordrecht, Holland, 1983.

[6] Matz, "Maximum Likelihood parameter estimation for the quartic exponential distributions, " Technometrics, 20, 475-484, 1978.

[7] Mohammad-Djafari A. et Demoment G., "Estimating Priors in Maximum Entropy Image Processing, " Proc. of ICASSP 1990, pp: 2069-2072

[8] Mohammad-Djafari A. et Idier J., "Maximum entropy prior laws of images and estimation of their parameters," Proc. of The 10th Int. MaxEnt Workshop, Laramie, Wyoming, published in Maximum Entropy and Bayesian Methods, T.W. Grandy ed., 1990. 


\section{Annex}

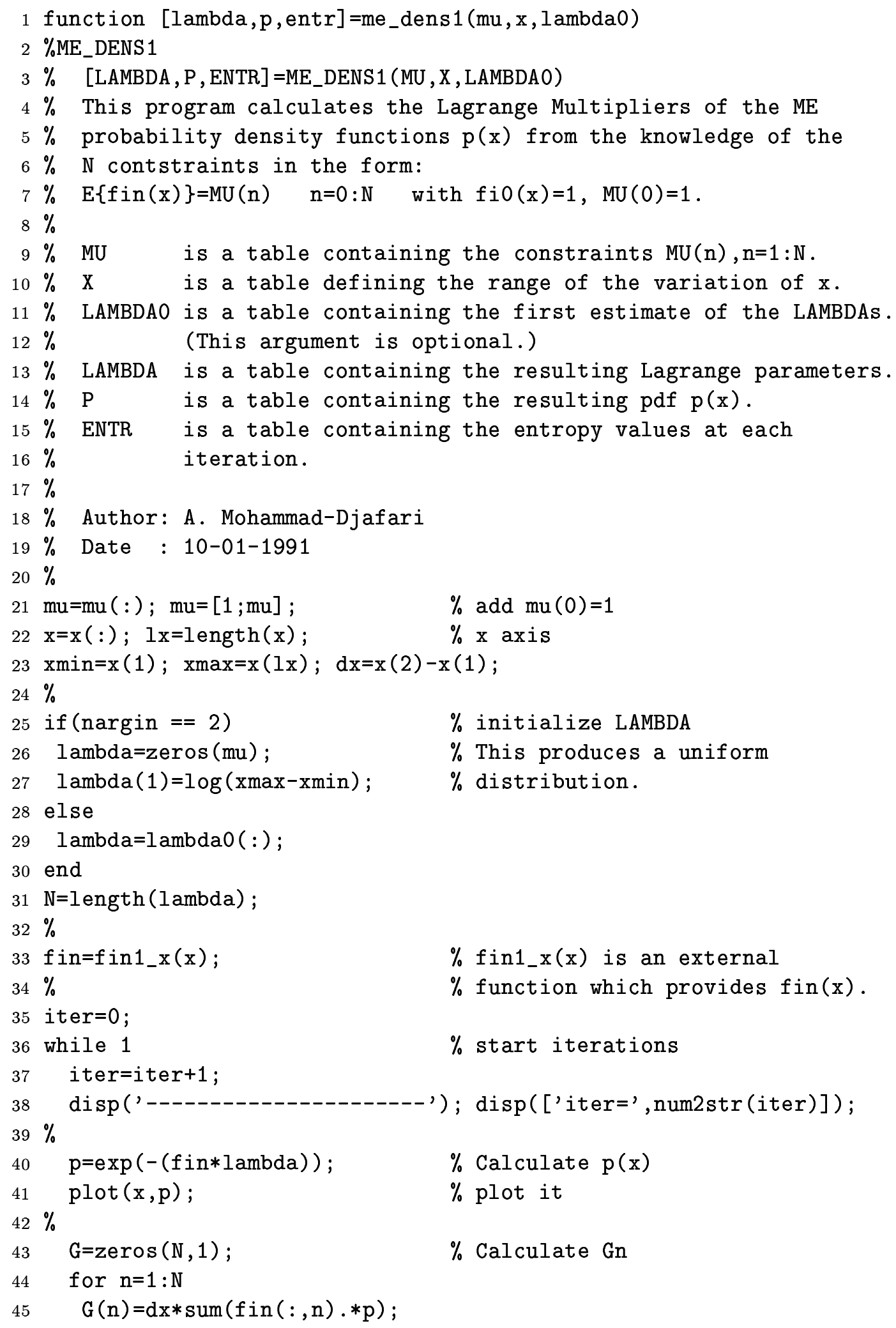




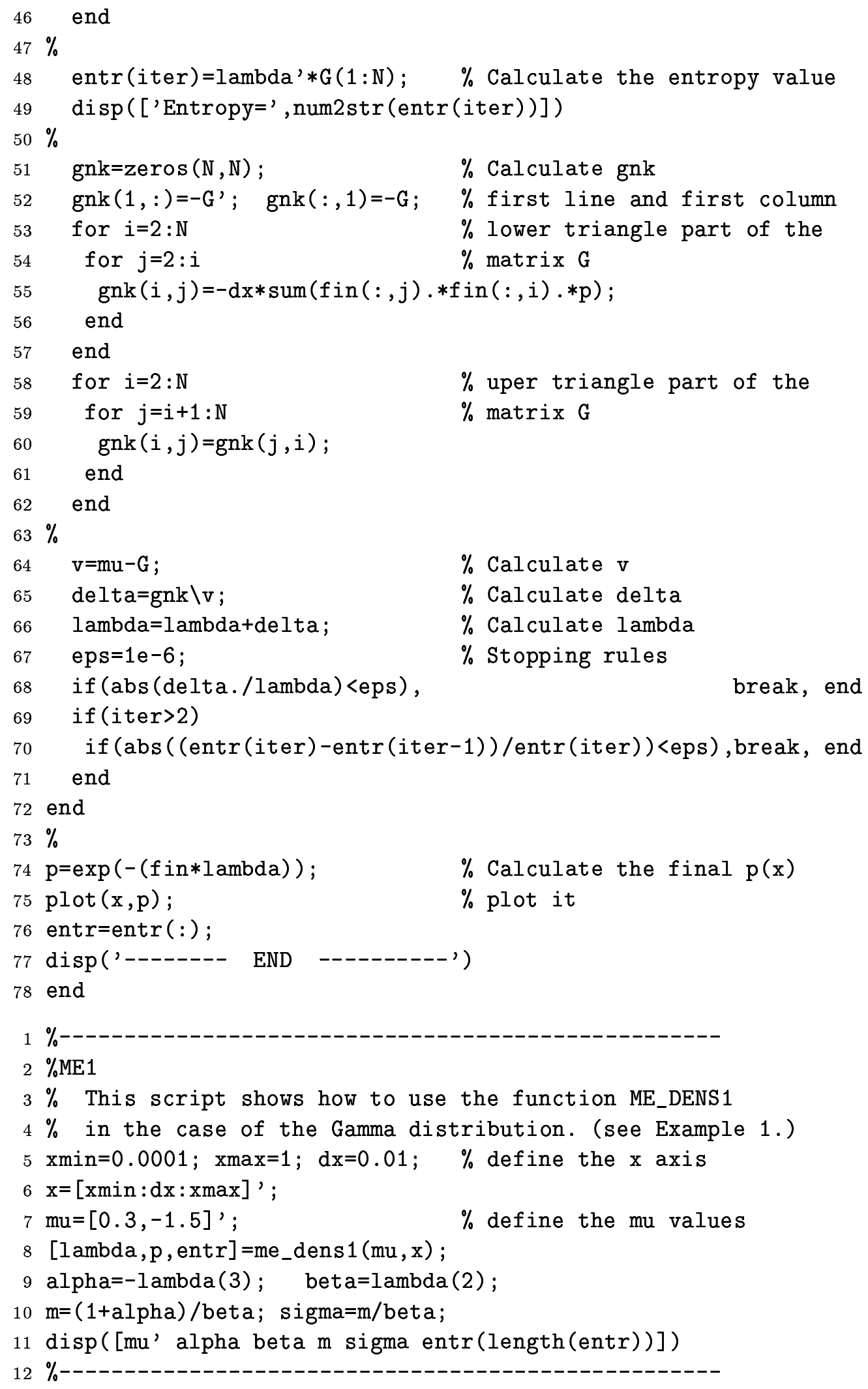




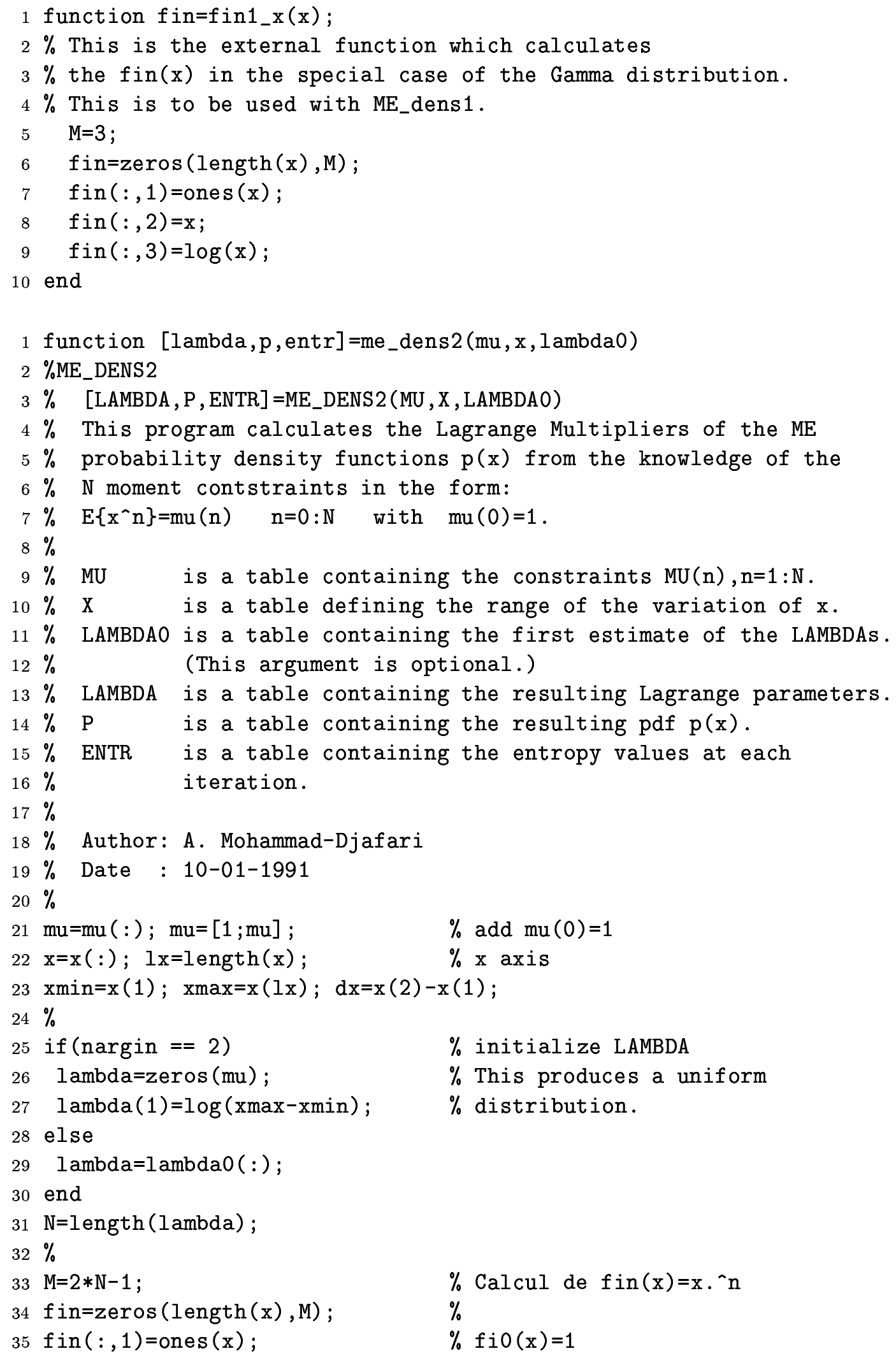




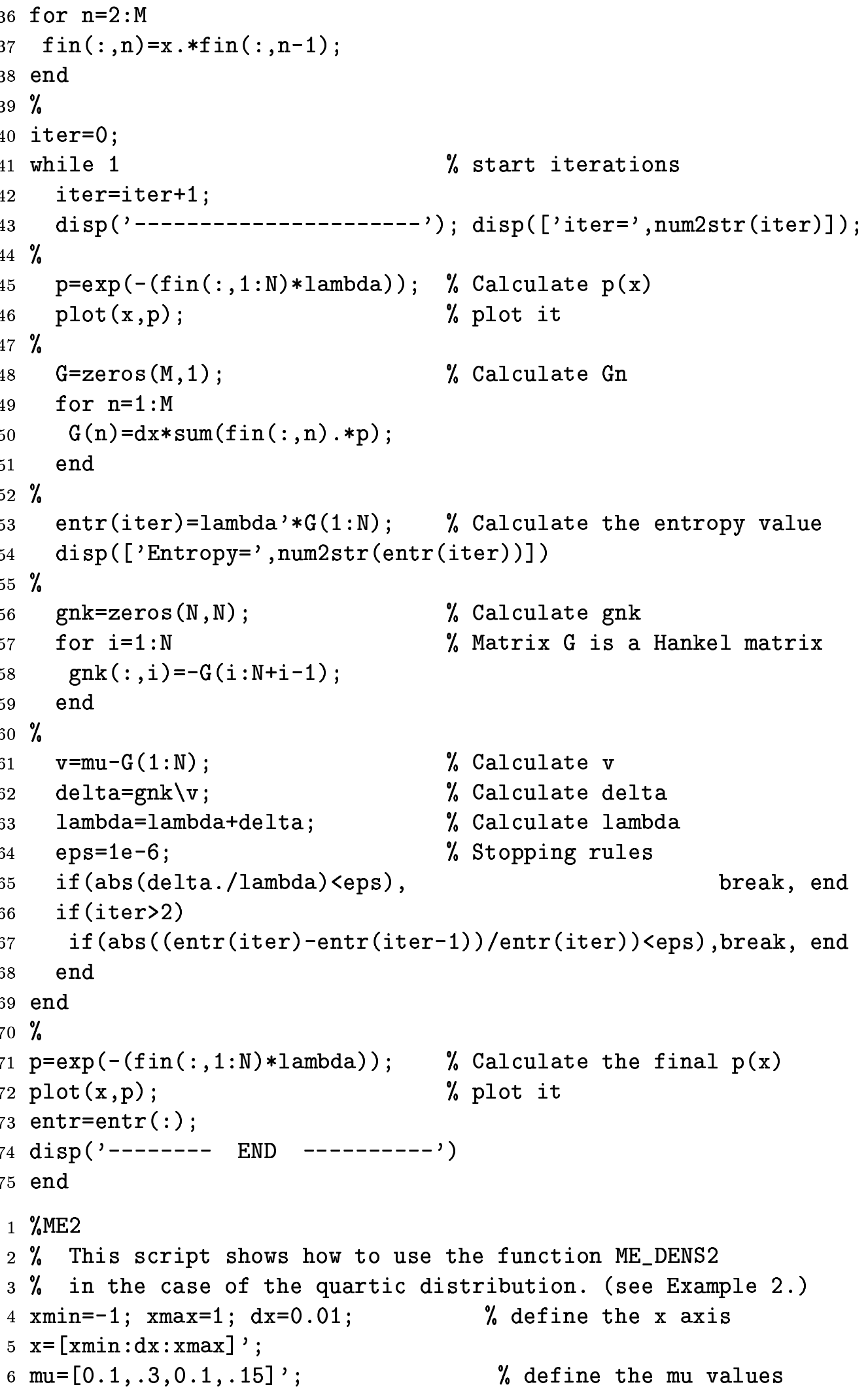




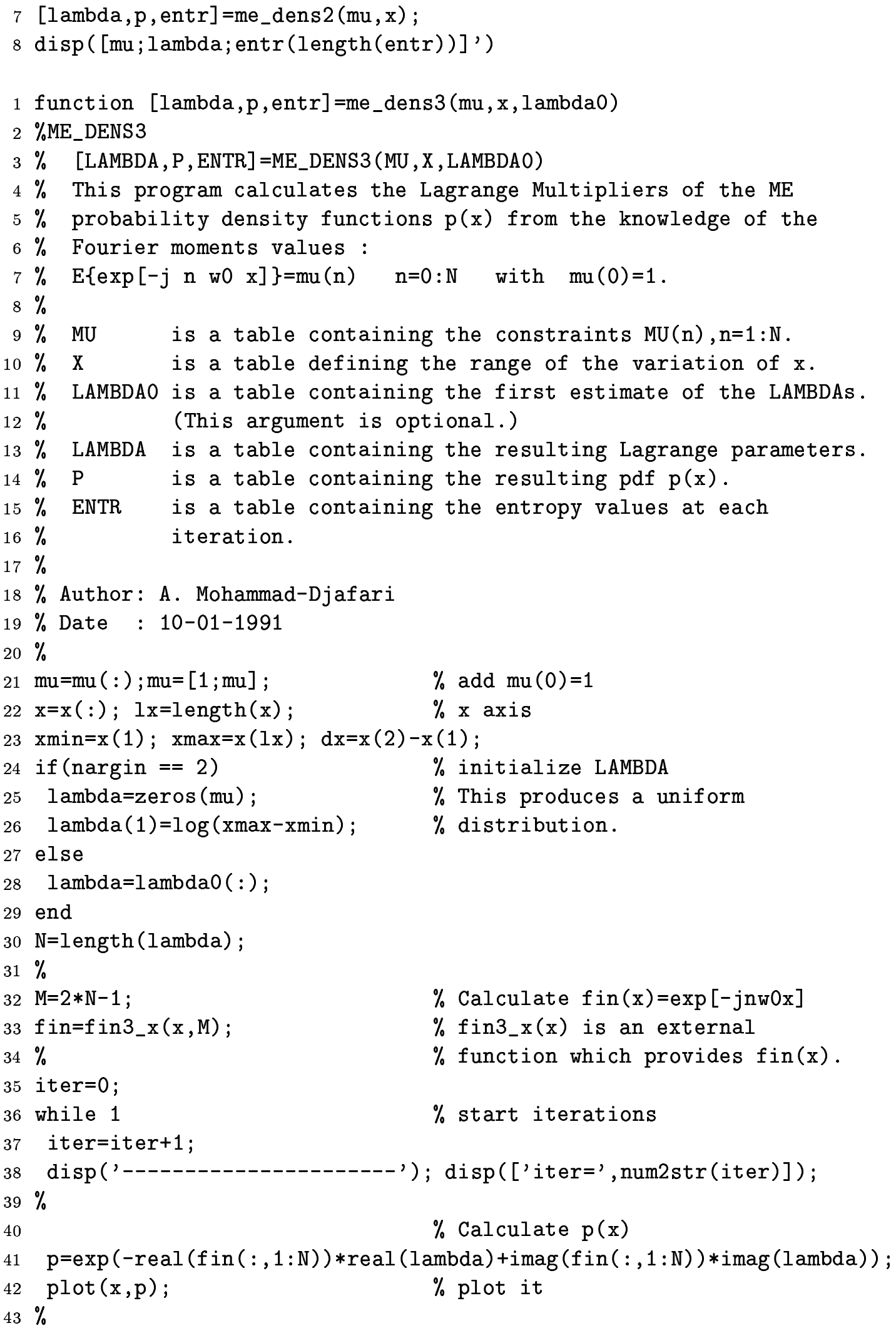




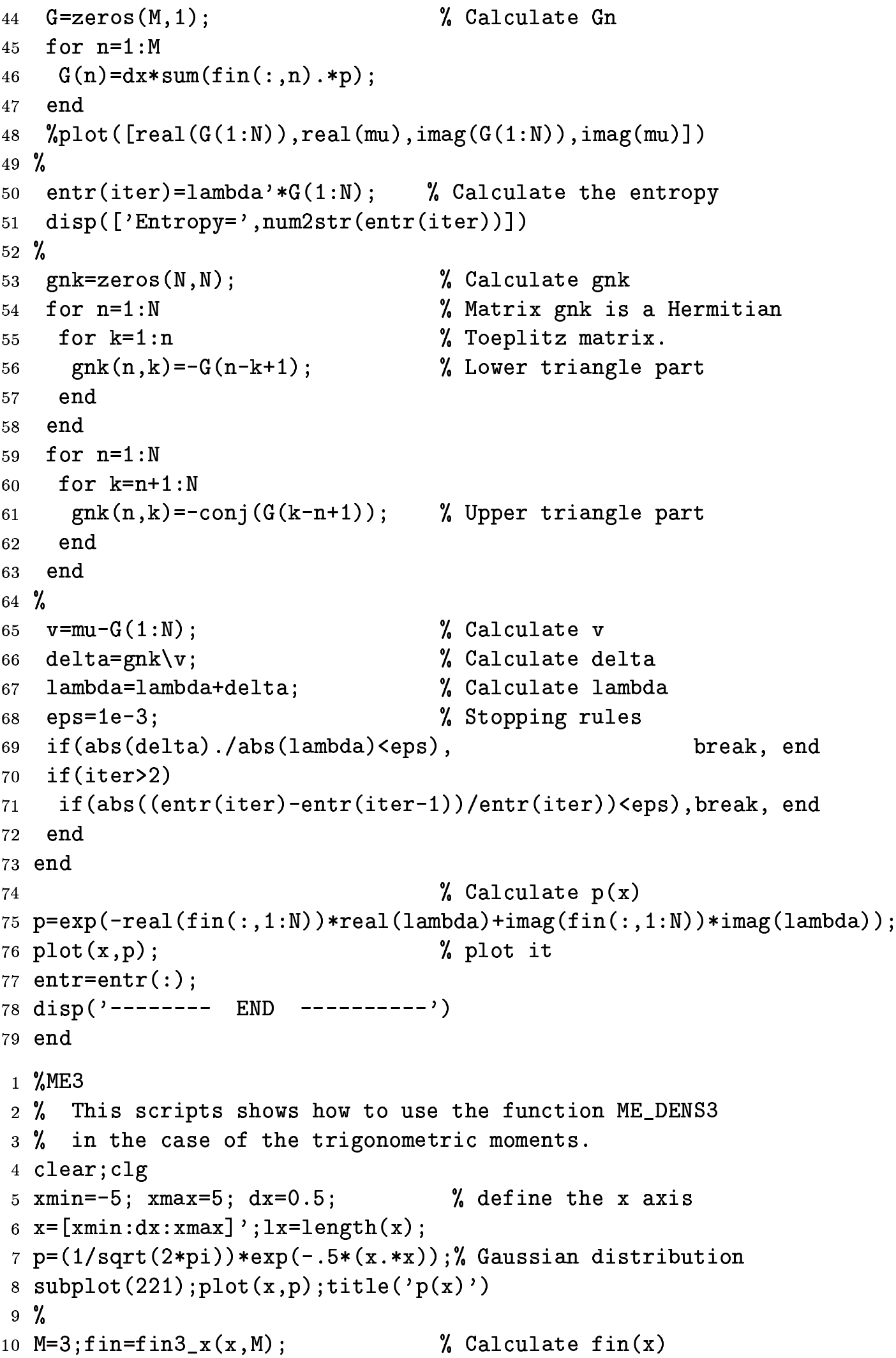




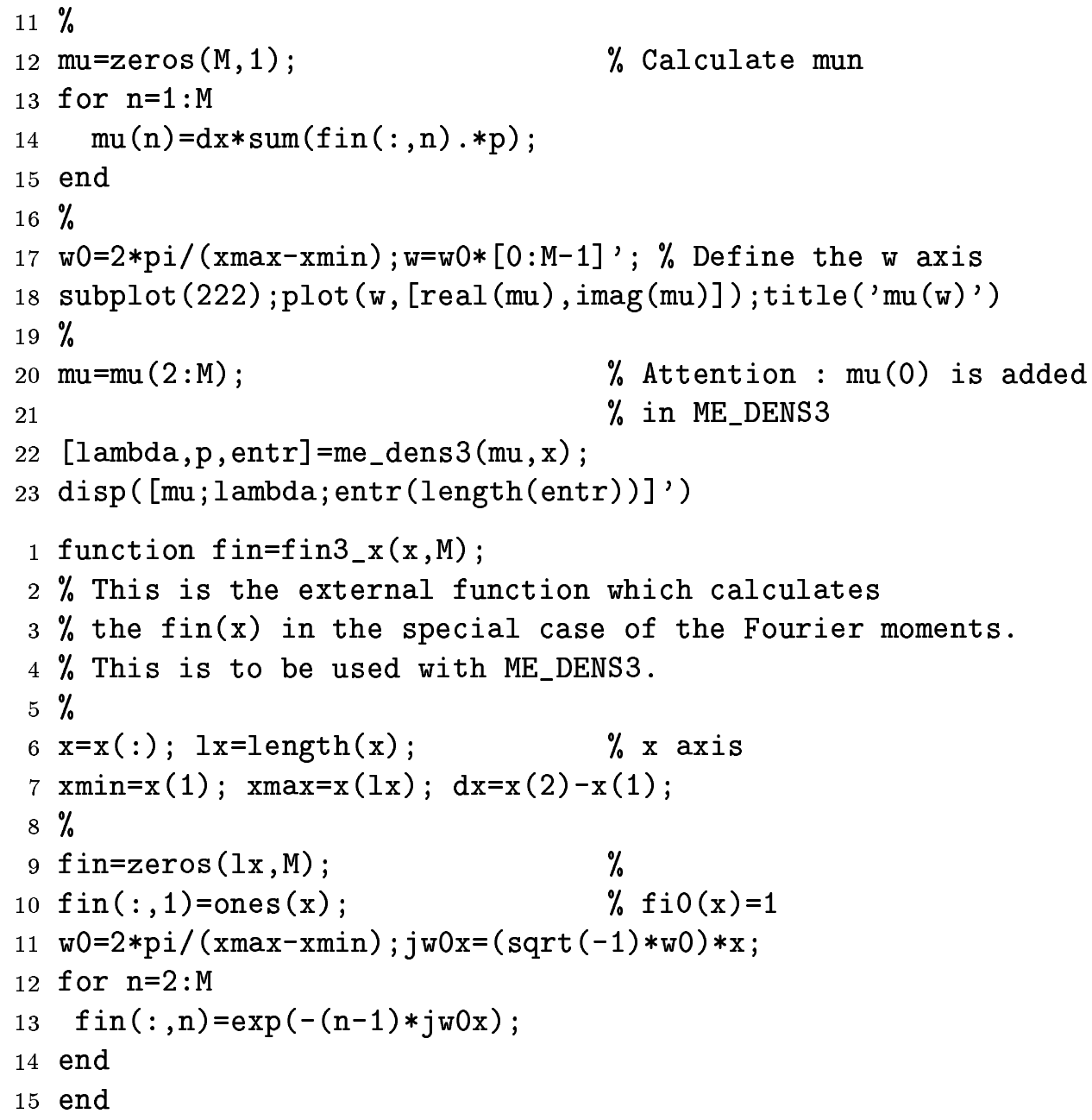

